\title{
Mobile Kommunikation der nächsten Generation
}

\author{
H. Malleck OVE, C. Mecklenbräuker
}

Online publiziert am 27. November 2012

(C) Springer-Verlag Wien 2012

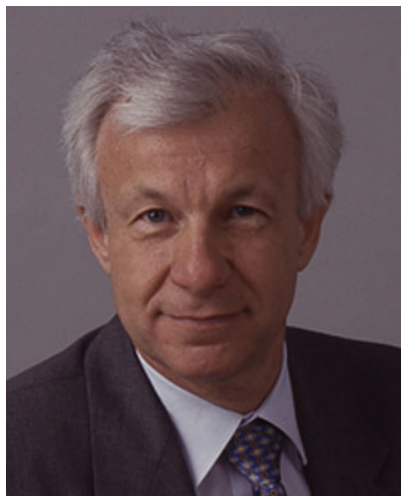

Helmut Malleck

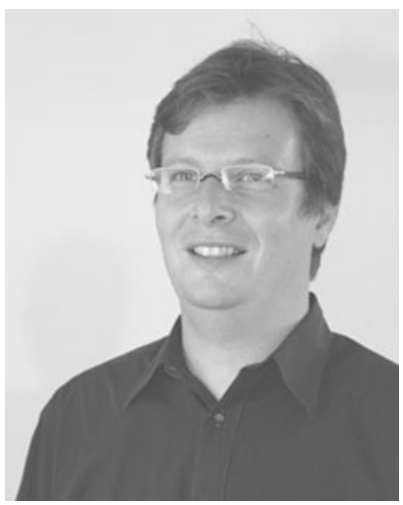

Christoph Mecklenbräuker

\begin{abstract}
Liebe Leserin und lieber Leser,

die vorliegende e\&i-Ausgabe ist - ebenso wie es das Informationstechnische Kolloquium im Mai 2012 an der Technischen Universität Wien bereits war - dem österreichischen Mobilfunkpionier Ernst Bonek zum 70. Geburtstag gewidmet. Der akademische Lehrer und Forscher ein Förderer des akademischen Nachwuchses, erkannte frühzeitig die Chancen der Mobilkommunikation. Als visionärer Gestalter prägte Professor Bonek langfristig die Geschicke des Forschungszentrums Telekommunikation Wien FTW auf Basis seines exzellenten theoretischen Wissens, mit Hartnäckigkeit und Durchhaltevermögen sowie mit Brückenschlägen von abstraktem Denken zur praktischen Umsetzung. Aktuelles Bestreben von Bonek ist es, durch kritisches Überlegen den Menschen die „HandyAngst"zu nehmen.
\end{abstract}

Der OVE mit seiner Verbandszeitschrift e\&i gratuliert Herrn Universitätsprofessor Dipl.-Ing. Dr. Ernst Bonek sehr herzlich zum Geburtstag und wünscht ihm weiterhin das Beste bei allen seinen Unternehmungen.

Mit den Beiträgen in dieser e\&i-Ausgabe wird für alle Leserinnen und Leser der Bogen gespannt von Antennen der nächsten Generation, Fehlerverschleierungsverfahren auf der Funkstrecke hin zu Diskussionen über Resourcen- und regulatorischen Fragen der Breitbandkommunikation. Sie finden dazu folgende Beiträge in dieser Ausgabe:

Antennen für die nächste Mobilfunk-Generation Vor 70 Jahren konzentrierten sich theoretische Untersuchungen auf die Feldverteilung bei Zylinderdipolen. Mittlerweile wurden enorme Fortschritte mit speziellen Antennenformen zu Antennengewinn und Bandbreite bei der Freiraum-Wellenausbreitung erzielt. Die Situation veränderte sich jedoch grundsätzlich mit sich zufällig bewegenden Einrichtungen und kleinen Antennenplattformen. Zukünftig werden bei Mobilkommunikation mehrere Antennen in einem Gerät untergebracht. Daraus ergeben sich neue Fragestellungen in Bezug auf Bandbreite, Wirkungsgrad und Korrelation, zu denen adaptive Lösungsansätze hier diskutiert werden.

Fehlerverschleierungsanalyse in H264/AVC-codierten Videosequenzen Die Fehlerverschleierungsanalyse bei fehlerbehafteten Funkstrecken rückt zunehmend in das Interesse, insbesondere, wenn keine Zweitübertragung von fehlerbehafteten Sequenzen möglich ist. Nach vergleichenden Leistungsanalysen verschiedener Verschleierungsverfahren mit weitgehend unbekannter Fehlerposition wird die analytische Behandlung der Verschleierung auf SliceEbene vorgestellt. Diese führt zu einem exakten mathematischen Modell. Weitere Verbesserungen auf Basis subjektiver Verfahren der visuellen Inspektion werden anhand von Simulationen verifiziert.

Mehr Ressourcen für mobiles Breitband: Technologien - Märkte - Regulation - Standardisierung Zur Beseitigung der Ressourcenknappheit wird zunächst eine weitere Steigerung der spektralen Effizienz und damit bessere Nutzung vorhandener Ressourcen im Zugangsnetz diskutiert. Alternativ dazu wird die Zuteilung von zusätzlichem Spektrum aus der Digitalen Dividende angeführt, was jedoch internationale Harmonisierungen voraussetzen würde. Als unerwartet einfacher Lösungsweg wird schließlich die Erhöhung der räumlichen Dichte der Zugangsknoten durch Einbindung von endnutzerbetriebenen so genannten "Small Cell"-Netzen vorgestellt.

Regulatorische Herausforderungen für die Migration von öffentlicher Telefonie ins Internet Kommunikationsdienstbetreiber verlagern Festnetz- und Mobilfunktelefonie ins Internet, wobei die Internettelefonie zumeist die öffentliche Telefonsysteme im Internet emuliert. Authentifizierung und Validierung als signifikante Herausforderung bleiben dabei oftmals unberücksichtigt. Eine Evolution zu separierten Funktionen, modularem Design und Implementierung von neuen IP-basierten Funktionen sowie eine Korrektur bisheriger Schwächen werden dargestellt.

Darüber hinaus finden Sie in dieser Ausgabe zwei Berichte, die sich unterschiedlichen Aspekten des mobilen Breitbandes widmen: „Evolution and deployment of VoLTE (Voice-over-Long-TermEvolution)" von Huawei sowie „Heterogeneous networks - meeting mobile broadband expectations with maximum efficiency", ein White Paper von Ericsson.

Malleck, Helmut, Dipl.-Ing. Dr., Arbeitskreis für technische Koordination für öffentliche Kommunikationsnetze und -dienste (AK-TK), Postfach 147, 1031 Wien, Österreich (E-Mail: helmut.malleck@oefeg.at); Mecklenbräuker, Christoph, Univ.-Prof. Dipl.-Ing. Dr.-Ing., Institute of Telecommunications, Technische Universität Wien, Gußhausstraße 25, 1040 Wien, Österreich 
Technologische Weiterentwicklungen ermöglichen uns mittlerweile, per Smartphone und Tablet Computer nicht nur mobile Sprachkommunikation, sondern vor wenigen Jahren noch unvorstellbar große Datenmengen zu empfangen und zu verarbeiten. Mobile Downloads von Musik-, Film- und Fernsehestreams sowie der Zugriff auf soziale Netzwerke sind praktisch möglich und werden für immer mehr Menschen alltägliche Selbstverständlichkeit. Herkömmliche mobile Netze stoßen dadurch an ihre Leistungsgrenzen. Daher investieren weltweit Mobilnetzbetreiber - trotz seit Jahren rückläufigem Trend bei Sprachdiensten - in den Netzausbau, insbesondere in flächendeckende LTEVersorgung. Die Global mobile Suppliers Association (GSA) berichtet in http://www.gsacom.com/news/gsa_352.php, dass in 38 Ländern insgesamt 80 kommerzielle LTE-Dienste verfügbar sind und bis Ende 2012 sich die Zahl auf 59 Länder und 144 kommerzielle LTE-Dienste erhöht. Globale mobile Breitbandkommunikation bleibt weiterhin ein spannendes Forschungs-Aufgabenfeld.

Viel Freude beim Lesen wünschen Ihnen
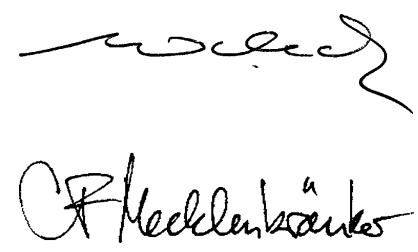